Reprod. Nutr. Dévelop., 1986, 26 (2 B), 729-730.

\title{
Effet du lait sur la régulation histaminergique de la sécrétion acide gastrique chez le rat
}

Ph. AVRIL, Florence HERVATIN, Evelyne MOREAU, B. GARZON, R. DUCROC, Ph. MILLET, J. P. GELOSO

Laboratoire de Différenciation fonctionnelle, Université Paris 7 2, place Jussieu, 75251 Paris Cedex 05.

Summary. In rats given a bovine milk diet for 4 days, basal acid secretion (SA) was significantly reduced in vitro and in vivo. Dose-response curves to histamine (HA) shifted to the right. As in suckling pups, ineffective concentrations of HA maximally stimulated SA in the presence of $\mathrm{H}_{2}$-receptor antagonists only. Milk was a potent inhibitor of HA-induced SA, possibly at the $\mathrm{H}_{2}$-receptor level.

Introduction. Chez le rat, le développement de la sécrétion acide (SA) gastrique qui s'instaure à la fin de la gestation, est caractérisé par une phase postnatale d'hyposécrétion qui coïncide avec le début de l'allaitement (1). Nous avons examiné la sensibilité de l'estomac de fœtus ( $21 \mathrm{j}$ post coïtum) et de nouveau-nés allaités (NN $5 \mathrm{j}$ post partum) à I'histamine (HA) en chambre de Ussing. L'effet du lait sur les mêmes paramètres a été étudié in vitro et in vivo sur des rats adultes nourris exclusivement au lait de vache.

Matériel et méthodes. Des rats mâles Wistar (150-200 g) sont alimentés à I'UAR 113 (Témoins). Les rats nourris au lait cru (NLC), au lait stérilisé entier (NLS) ou au lait stérilisé écrémé (NLE) sont mis à jeun $16 \mathrm{~h}$ avant de recevoir l'alimentation lactée pendant 4 jours. In vitro : les estomacs de fœtus et de NN ainsi que les muqueuses fundiques de rats adultes sont tendus entre les deux compartiments d'une chambre de Ussing. Le flux net d'ion $\mathrm{H}^{+}\left(\mathrm{JH}^{+}\right.$net) es: mesuré à pH-stat 7,0 par titration dans la chambre avec $\mathrm{NaOH} 0,01 \mathrm{M}$. L'HA, I'HA et la cimétidine (CIM) ou le dibutyryl (db) AMPc sont introduits du côté séreux après avoir mesuré $\mathrm{JH}^{+}$net basal. $\mathrm{JH}^{+}$net est déterminé toutes les 10 min et exprimé en $\mu \mathrm{Eq} / \mathrm{h} . \mathrm{cm}^{2}$ (SA max : $200 \pm 20 \mathrm{~min}$ ). In vivo : les rats sont opérés suivant Ghosh et Schild (1958) modifié par Lai (1964) : estomac perfusé par $\mathrm{NaCl} 0,9 \%$ à $37^{\circ} \mathrm{C}(0,7 \mathrm{ml} / \mathrm{min})$. L'acidité $\left(\mu \mathrm{Eq} \mathrm{h}{ }^{+} / 10 \mathrm{~min}\right)$ est titrée à $\mathrm{pH} 7$. La SA basale est mesurée avant administration de $\mathrm{HA}(0,25-2,5 \mathrm{mg} / \mathrm{kg}$, i.p.) seule ou avec CIM $(0,35-1,4 \mathrm{mg} / \mathrm{kg}) \mathrm{SA} \max : 30 \pm 10 \mathrm{~min}$.

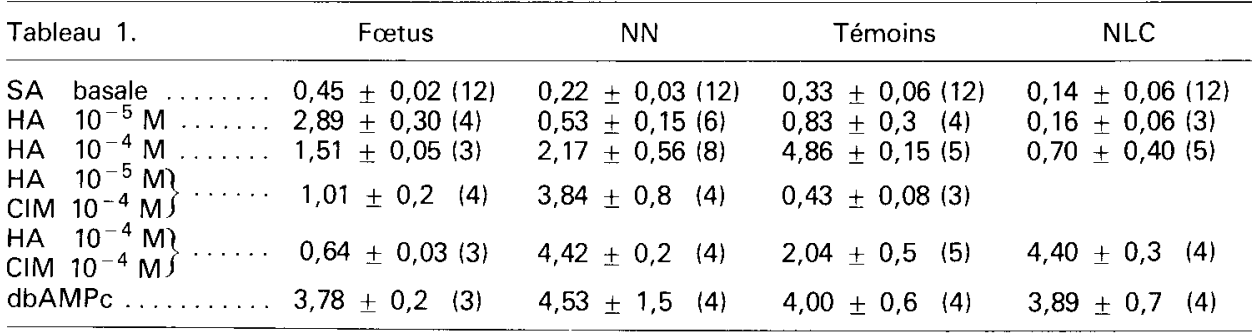

Moyenne \pm écart-type ; entre parenthèses : nombre de cas 
Résultats/Discussion. In vitro (tabl. 1), on observe une réduction significative de $\mathrm{JH}^{+}$net basal, chez le NN comme chez le NLC par rapport aux fœtus et aux témoins. Dans ces deux groupes alimentés au lait, la SA maximale est obtenue : 1) à la concentration 10 fois supérieure d'HA ; 2) paradoxalement en présence de CIM ou, 3) en présence de dbAMPc $10^{-4} \mathrm{M}$. Chez les rats NLE la sensibilité à I'HA et à la CIM n'est pas différente de celle des témoins.

In vivo, on observe également une réduction significative de la SA basale chez les NLC. Les courbes dose-réponses à $\mathrm{HA}$ (fig. 1) indiquent clairement que chez le NLC, l'effet de HA est inhibé de façon compétitive. Comme in vitro, CIM + HA a l'effet paradoxal de permettre une réponse maximale. La résistance à HA et l'effet de CIM suggèrent une diminution de la capacité de HA d'interagir efficacement avec le système récepteur $\mathrm{H}_{2}$ /adenylate cyclase, comme cela est observé au cours du développement (2). La réponse à $\mathrm{HA}+\mathrm{CIM}$ chez les rats nourris au NLE n'est pas modifiée par rapport à celle obtenue chez les rats témoins (tabl. 2).

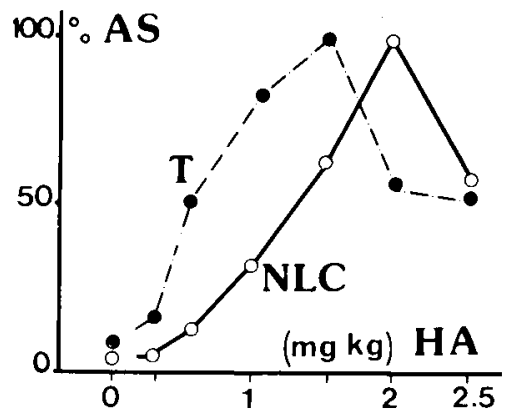

FIG. 1. - Courbes dose-réponses à HA chez les rats $T(0)$ et $N L C(O)$. $100 \%=$ réponse maximale.

\begin{tabular}{|c|c|c|c|c|}
\hline Tableau 2. & Témoins & NLC & NLS & NLE \\
\hline SA basale. & $0,61 \pm 0,07(13)$ & $0,34 \pm 0,06(10)$ & $1,09 \pm 0,18(8)$ & $1,07 \pm 0,30(6)$ \\
\hline $\mathrm{HA}(0,5 \mathrm{mg} / \mathrm{kg})$ & $3,52 \pm 0,32(5)$ & $0,85 \pm 0,11(5)$ & $1,51 \pm 0,28(3)$ & $2,82 \pm 0,04(3)$ \\
\hline $\mathrm{HA}+\mathrm{CIM} \ldots$ & $0,88 \pm 0,13(4)$ & $1,85 \pm 0,25(5)$ & $0,90 \pm 0,04(3)$ & $1,02 \pm 0,16(3)$ \\
\hline
\end{tabular}

Moyenne \pm écart-type ; entre parenthèses : nombre de cas.

Chez les rats recevant un lait stérilisé non écrémé (NLS), la réponse à HA est réduite de $45 \%$ par rapport à celle obtenue chez les rats NLE, mais l'effet inhibiteur de $\mathrm{CIM}$ sur la réponse à $\mathrm{HA}$ est conservé. Ces résultats indiquent que le lait possède une puissante activité modulatrice de la SA. Les facteurs directement ou indirectement responsables de cette activité sont à rechercher dans la fraction lipidique et/ou parmi les molécules thermosensibles du lait.

Conc/usion. Le lait possède donc, en plus de son pouvoir tampon, des propriétés antisécrétoires qui rendent compte, chez le rat, du développement diphasique de la sécrétion acide gastrique au cours de la période néonatale.

(1) Garzon B., Ducroc R., Onolfo J. P., Desjeux J. F., Geloso J. P., 1982. Am. J. Physiol., 242, G111-115.

(2) Gespach C., Cherel Y., Rosselin G., 1984. Am. J. Physiol., 277, G231-239.

(3) Ghosh M., Schild H. O., 1958. Br. J. Pharmacol., 13, 54-61.

(4) Lai K. S., 1964. Gut, 5, 327-333. 